Mit at i onal anal ysi s of human RNA pol ymer ase II subunit 5 (RPB5): The resi dues critical for i nt er act i ons wi th TFI I F subunit RAP30 and hepat it is $B$ vi rus $X$ protei $n$

\begin{tabular}{|l|l|}
\hline 著者 & $\begin{array}{l}\text { Le Thi Thu Thuy, Zhang Shi } \mathrm{j} \text { un, Hayashi } \\
\text { Naoyuki, Yasukawa Nami, Del ger maa Luvsanj av, } \\
\text { Mur akami Sei shi }\end{array}$ \\
\hline 著者別表示 & 林 直之, 村上 清史 \\
\hline $\begin{array}{l}\text { j our nal or } \\
\text { publ i cat i on ti l l e }\end{array}$ & Journal of Bi ochem st ry \\
\hline vol une & 138 \\
\hline nunber & 3 \\
\hline page r ange & $215-224$ \\
\hline year & $2005-09-01$ \\
\hline URL & ht t p: //doi . or g/10. 24517/00027528 \\
\hline
\end{tabular}




\title{
Mutational Analysis of Human RNA Polymerase II Subunit 5 (RPB5): The Residues Critical for Interactions with TFIIF Subunit RAP30 and Hepatitis B Virus $X$ Protein
}

\author{
Thi Thu Thuy Le, Shijun Zhang, Naoyuki Hayashi, Mami Yasukawa, \\ Luvsanjav Delgermaa and Seishi Murakami* \\ Department of Molecular Oncology, Cancer Research Institute, Kanazawa University, Takara-machi 13-1, \\ Kanazawa 920-0934 \\ Received April 14, 2005; accepted May 2, 2005
}

\begin{abstract}
RNA polymerase II (RNAPII) subunit 5 (RPB5) is positioned close to DNA downstream of the initiation site and is the site of interaction with several regulators. Hepatitis $B$ virus $X$ protein $(\mathrm{HBx})$ binds the central part of RPB5 to modulate activated transcription, and TFIIF subunit RAP30 interacts with the same part of RPB5 that is critical for the association between TFIIF and RNAPII. However the residues necessary for these interactions remain unknown. Here we report systematic mutagenesis of the central part of RPB5 using two-step alanine scanning libraries to pinpoint critical residues for its binding to RAP30 in the TFIIF complex and/or to HBx, and identified these residues in both mammalian cells and in an in vitro binding assay. Four residues, F76, I104, T111 and S113, are critical for both TFIIF- and HBx-binding, indicating the overlapping nature of the sites of interaction. In addition, V74 and N98 are required for HBx-binding, and T56 and L58 are needed for RAP30-binding. Interestingly the residues exposed to solvent, T111 and S113, are very close to the DNA, implying that two factors may modulate the interaction between DNA and RPB5.
\end{abstract}

Key words: alanine scanning, coactivation, HBx, RAP30, RPB5, TFIIF.

Abbreviations: RPB5, RNA polymerase subunit 5; HBx, hepatitis B virus X protein; RNAPII, RNA polymerase II; RMP, RPB5-mediating protein; aa, amino acid.

Transcription is the primary regulatory step of eukaryotic gene expression and is carried out by DNA-dependent RNA Polymerase II (RNAPII) along with general transcription factors (GTFs), transcription factors, and cofactors (1-7). RNAPII is the ultimate target of transcription factors and cofactors acting directly or indirectly to modulate transcription. During the processes of transcription, RNAPII changes partners or complexes with which it interacts by altering its conformation. In this context, several RNA polymerase subunits have recently been reported to interact with these regulators (5). RNAPII consists of 12 subunits that are well conserved from yeast to human. Crystal structures and cryoelectron microscopy have solved a subset of RNAPII transcription complexes $(3,7-12)$ that provide deep insight into the molecular basis of RNAPII transcription.

RNA polymerase II subunit 5 (RPB5) is part of the lower jaw of RNAPII, and the exposed domain of RPB5 serves in interactions with transcriptional regulators including Hepatitis $\mathrm{B}$ virus $\mathrm{X}$ protein ( $\mathrm{HBx})$, TFIIB, Tip120, TFIIF subunit RAP30 and RMP/URI (3, 8, 1319). Human RPB5 consists of an exposed domain (aa 1 to 139) and an embedded domain (aa 140 to 210), which are well conserved between yeast and human (20-22). There is no interdomain interaction in the crystal models of

"To whom correspondence should be addressed. Phone: +81-76-2652731, Fax: +81-76-234-4501, E-mail: semuraka@kenroku.kanazawau.ac.jp
RPB5, implying the flexible nature of the exposed domain relative to the embedded domain $(8,21)$. The exposed domain seems to comprise two subdomains, the $\mathrm{N}$-terminal part (aa 1 to 47 ), and the central part of RPB5 (aa 57 to 139), with a short loop between the subdomains. The N-terminal part is outside of the lower jaw, and the central part of RPB5 is mostly inside the jaw and close to the DNA downstream of the initiation site. The former is critical for TFIIB-binding and the latter harbors the sites of interaction with RAP30 and HBx, and probably RMP/ $\operatorname{URI}(8,13,15,16,18,19)$.

TFIIF, a heterotetramer of RAP74 and RAP30, is a unique general transcription factor that functions in initiation, elongation, and recycling of transcription $(4,18$, $23,24)$. TFIIF binds directly to RNAPII and also helps to recruit the enzyme to the promoter $(14,25-28)$. TFIIF can induce further bending and wrapping of the promoter DNA against the mobile clamp of RNAPII during the formation of the preinitiation complex $(11,29-32)$. The direct interaction between the central region of RAP30 and the central part of RPB5 has been reported to contribute to the association between TFIIF and RNAPII (18), although other interactions between TFIIF and RNAPII may occur during transcription (11).

$\mathrm{HBx}$ is a multifunctional viral regulator protein of Hepatitis B Virus that coactivates activated, but not basal, transcription in vivo and in vitro $(17,33,34)$. The direct interactions of RPB5 with HBx and TFIIB are critical for the coactivating ability of $\operatorname{HBx}(13,15,17)$, which augments HBV replication positively (35) and modulates 
gene expression of cells. Previously we identified a novel cellular protein, RPB5-mediating protein (RMP) (16) or Unconventional RPB5-interacting protein (URI) (36), which modulates transcription as a corepressor through binding to RPB5, and counteracts transcriptional modulation by $\mathrm{HBx}(16)$.

It remains unclear whether the sites of RPB5 targeted by RAP30 and HBx are different or redundant. Here we specified the residues critical for the interactions with HBx and/or RAP30 by two-step alanine scanning with mutant libraries of RPB5. Four residues, F76, I104, T111 and S113, were found to be essential for binding to the two factors, indicating the overlapping nature of the interactions.

\section{MATERIALS AND METHODS}

Plasmid Constructions-The plasmids pNKFLAG and pNKGST derived from pSG5UTPL are FLAG-tagged and GST-tagged mammalian expression vectors, respectively, as reported. The plasmids pGENK1 and pGENKS are bacterial expression vectors for GST-fused proteins (13, 16). The full-length coding region of human RPB5 (XAP4) (1-210) was prepared as reported (13). Mutagenesis was carried out by a splicing PCR method using AGCGAATTCCATGGACGACGAGGAGGAG or AGTAGATCTCTACTGCACCAG together with mutated oligonucleotide primers (sequences available upon request). An alaninescanning method was used to construct RPB5 clustered or single alanine substitution mutants to minimize the effects of substituted amino acid residues $(15,37)$. The positions of clustered mutants of RPB5 are shown in Fig. 1. The target sequence of 7 amino acid residues was changed to AAASAAA for practical reasons in all clustered mutants $(\mathrm{cm})$. The second series of $\mathrm{cm}$ harboring AAASA [cm I(s)] (spanning from the 1st to 5th residue of the defining $\mathrm{cm}$ ) and SAAA [cm II(s)] (spanning from the 4 th to 7 th residue of the defining $\mathrm{cm}$ ) were constructed using the NheI site-encoding SA sequence. Then single alanine substitution mutants were constructed as follows: (i) if cm I(s) is defective, cm II(s) has no effect, first three residues will be targeted to point mutant scanning; (ii) if $\mathrm{cm} \mathrm{I}(\mathrm{s})$ has no effect, $\mathrm{cm} \mathrm{II(s)} \mathrm{is} \mathrm{defective,} \mathrm{last} \mathrm{two}$ residues will be targeted to point mutant scanning; (iii) if both $\mathrm{cm} \mathrm{I}(\mathrm{s})$ and $\mathrm{cm} \mathrm{II(s)} \mathrm{are} \mathrm{defective,} \mathrm{all} \mathrm{seven} \mathrm{residues}$ will be targeted to point mutant scanning. In the point substitution mutants, the target residue was replaced with alanine (A) in a two-step PCR scanning. All sequences encoding full-length mutants of RPB5 were inserted into the EcoRI and $B g l \mathrm{II} / B a m \mathrm{HI}$ sites of pNKFLAG, pNKGST, pGENKS, and pYFLAG to construct various expression vectors. All of the constructs were sequenced by the dideoxy method using Taq sequencing kits and a DNA sequencer (370A; Applied Biosystems Inc. Co. Ltd.).

The plasmids pGST-RAP30 and His-ET-RAP74 were gifts from R.G. Roeder. The truncation mutant of RAP30 cDNA, RAP30/d4, encodes the initiation codon followed by amino acids 101 to 249 , as reported (18).

The Escherichia coli histidine-tagged protein expression plasmid pLHis was derived from pET11d, by replacing the NdeI-BamHI fragment with the annealed complementary oligonucleotides TATGAATTCCATGAA-

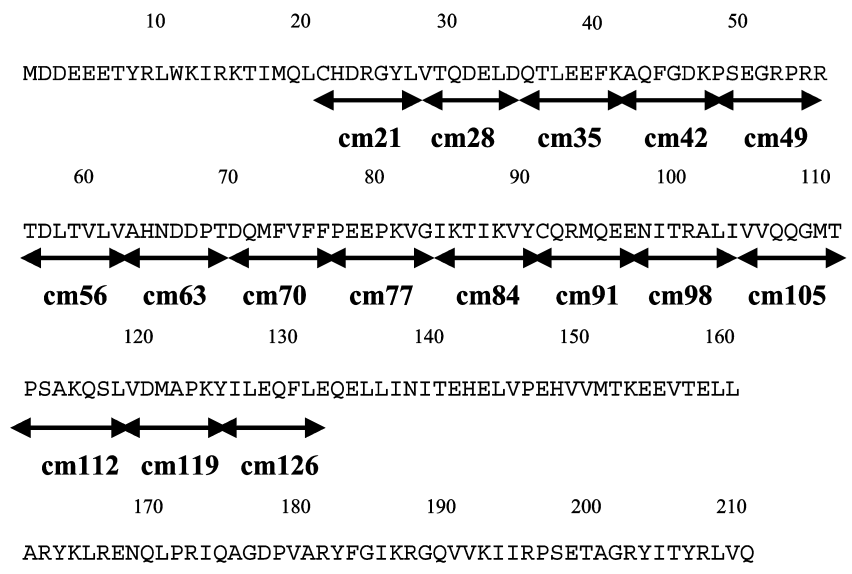

Fig. 1. Clustered substitution mutants (cm) of human RPB5. The amino acid sequence of human RPB5 and a mutant library of human RPB5 were constructed. The target of 7 amino acid residues was changed to AAASAAA. Names of clustered mutants $(\mathrm{cm})$ are shown under two-headed arrows with numbers corresponding to the number of the first of the 7 amino acid residues substituted with AAASAAA in the human RPB5 open reading frame.

GCTTGGATC and GATCCAAGCTTCATGGAATTCATA, to introduce EcoRI, HindIII, and BamHI digestion sites. The plasmid pLHis-HBx was constructed by inserting the coding sequence of wild type HBx into the EcoRI and Bam HI sites of pLHis as reported (33).

Preparation of Recombinant Proteins-GST-fused proteins were expressed in $E$. coli by induction with $0.4 \mathrm{mM}$ isopropyl-D-thiogalactopyranoside at $30^{\circ} \mathrm{C}$ for $3 \mathrm{~h}$. The cells were harvested and sonicated in PBST buffer (phosphate-buffered saline containing $1 \mathrm{mM}$ DTT and $1 \%$ Triton X-100) $(16,18)$. After centrifugation of the sonicated lysate, the supernatant was passed through DEAE Sepharose, and GST-fused proteins were recovered with glutathione-Sepharose 4B beads (Amersham Biosciences, Inc.) at room temperature for $1 \mathrm{~h}$. The beads were sedimented, washed four times with an excess amount of PBST buffer, and then eluted with $10 \mathrm{mM}$ of reduced glutathione in $50 \mathrm{mM}$ Tris- $\mathrm{HCl}$ ( $\mathrm{pH}$ 8.0). The eluted solution was dialyzed against buffer B [100 mM Tris-HCl $(\mathrm{pH}$ 8.0), $150 \mathrm{mM} \mathrm{NaCl}$, and $1 \mathrm{mM}$ DTT], then divided into aliquots and stored at $-80^{\circ} \mathrm{C}$.

The His-tagged $\mathrm{HBx}$ protein was expressed in BL21 (DE3)/pLys using $0.7 \mathrm{mM}$ isopropyl-D-thiogalactopyranoside for $3 \mathrm{~h}$ at $27^{\circ} \mathrm{C}$. The cell pellet was harvested and washed, and resuspended in precleared buffer [50 $\mathrm{mM}$ sodium phosphate ( $\mathrm{pH} 8.0$ ), $500 \mathrm{mM} \mathrm{NaCl}, 10 \%$ glycerol, $1 \mathrm{mM}$ phenylmethylsulfonyl fluoride, $10 \mathrm{mM} \beta$ mercaptoethanol, and $1 \%$ Triton $\mathrm{X}-100]$. The suspension was sonicated on ice and centrifuged at $12,000 \times g$ for 15 min. The pellet was suspended in denaturing binding buffer (100 mM sodium phosphate, $10 \mathrm{mM}$ Tris- $\mathrm{HCl}$, and $8 \mathrm{M}$ urea) with the $\mathrm{pH}$ adjusted to 8.0 using $\mathrm{HCl}$. The lysate was centrifuged at $12,000 \times \mathrm{g}$ for $5 \mathrm{~min}$, and the cleared lysate was subjected to affinity binding to nickel resin (Qiagen) preequilibrated with denaturing binding buffer. The resin was washed once with denaturing binding buffer then several times with denaturating buffer mixed with increasing amounts of bridge buffer $(100 \mathrm{mM}$ sodium phosphate, $10 \mathrm{mM}$ Tris-HCl, $2 \mathrm{mM}$ DTT, $1 \mathrm{M}$ 
Fig. 2. Amino acid sequences of RPB5 critical for HBxbinding as analyzed in vivo and in vitro. (A-C) In vivo assays: COS1 cells were transfected with pNKGST-HBx alone in the absence of pNKFLAGRPB5 (mock) (lane 1), or transiently cotransfected with the mammalian expression vectors pNKGST and pNKFLAG-RPB5 wild type (WT) (lane 2) or pNKGST-HBx and pNKFLAGRPB5 wild type (WT) or different $\mathrm{cm}$ RPB5 as indicated at the top (lanes 3 to 11). (A) Total lysates were separated by SDS-PAGE and subjected to Western blot analysis with $\alpha$-FLAG monoclonal antibody (input). (B) Cell lysates were pull-downed with glutathione resin. After washing, the bound proteins were detected with an $\alpha$-FLAG monoclonal antibody. (C) The nitrocellulose membranes used for Western blot analysis with the $\alpha$-FLAG antibody were reprobed with an $\alpha$ GST antibody. (D and E) In vitro assays: (D) Purification of bacterially expressed GST, GST-RPB5 wild type (WT) or different clustered mutants as indicated. The GST (lane 1) and GST-RPB5 WT (lane 2) or mutant RPB5 (lanes 3 to 10) proteins were expressed in E. coli and purified. Between 0.4 and $1 \mu \mathrm{g}$ of each purified protein was analyzed by SDS-PAGE and stained with Coomassie Brilliant Blue. (E) Interaction of RPB5 and $\mathrm{HBx}$ proteins in vitro. Approximately $1 \mu \mathrm{g}$ of bacterial GST or bacterial recombinant GST-fused RPB5 wild type (WT) or cm proteins was immobilized on glutathione resin and incubated with $0.2 \mu \mathrm{g}$ of His-HBx protein in GBT buffer for $2 \mathrm{~h}$ at $4^{\circ} \mathrm{C}$. Pulldown assays and Western blot analysis were carried out with an $\alpha$-His monoclonal antibody. Input (lane 1 ) and lanes 2 to 11 showed before and after the pull-down assay, respectively. Each experiment was repeated at least three times and the data were reproducible.

$\mathrm{NaCl}$, and $2 \%$ Triton $\mathrm{X}-100)$ to renature the protein, and finally washed with bridge buffer. The bound His-HBx was eluted with native elution buffer $[50 \mathrm{mM}$ sodium phosphate (pH 8.0), $500 \mathrm{mM} \mathrm{NaCl}, 10 \%$ glycerol, $1 \mathrm{mM}$ phenylmethylsulfonyl fluoride, $10 \mathrm{mM} \quad \beta$-mercaptoethanol, 1\% Triton X-100, and $500 \mathrm{mM}$ imidazole]. Eluted solutions were combined and dialyzed against buffer C [20 mM Tris-HCl (pH 7.5), $150 \mathrm{mM} \mathrm{NaCl}, 10 \%$ glycerol, $1 \mathrm{mM}$ DTT, and $0.1 \%$ Triton X-100], and stored at $-80^{\circ} \mathrm{C}$ in aliquots.

FLAG-tagged proteins were expressed in BL21 by induction with $0.4 \mathrm{mM}$ isopropyl-D-thiogalactopyranoside at $30^{\circ} \mathrm{C}$ for $3-6 \mathrm{~h}$. The cells were harvested and sonicated in $50 \mathrm{mM}$ Tris- $\mathrm{HCl}(\mathrm{pH} 8.0), 150 \mathrm{mM}$ $\mathrm{NaCl}$, and $0.1 \%$ Triton X-100. After centrifugation, the supernatant was stored at $-80^{\circ} \mathrm{C}$. FLAG-tagged proteins were purified by incubating the supernatant with anti-
GST pull-down

IB:Anti-FLAG

IB:Anti-FLAG

IB:Anti-GST

CBB-staining

IB:Anti-His

FLAG M2 resin (Kodak Scientific Imaging Systems) and washing several times. The bound proteins were eluted with buffer containing FLAG peptide $[0.2 \mathrm{mg} / \mathrm{ml}$ FLAG peptide, $50 \mathrm{mM}$ Tris- $\mathrm{HCl}(\mathrm{pH} 8.0)$, and $150 \mathrm{mM} \mathrm{NaCl}$. Eluted solutions were combined and dialyzed.

Antibodies-Anti-FLAG M2 and anti-His monoclonal antibodies were purchased from Kodak Science Imaging Systems. Anti-GST monoclonal antibody was obtained from ZYMED Laboratories.

In Vitro GST Resin Pull-Down Assays-Approximately $1 \mu \mathrm{g}$ of GST or GST-fused protein immobilized on $20 \mu \mathrm{l}$ of glutathione-Sepharose 4B preblocked in $1 \%$ bovine serum albumin was incubated with $0.2 \mu \mathrm{g}$ of FLAG-tagged proteins or $0.5 \mu \mathrm{g}$ of His-tagged proteins in $0.5 \mathrm{ml}$ of GBT buffer [10\% glycerol, $50 \mathrm{mM}$ Hepes- $\mathrm{NaOH}$ ( $\mathrm{pH} 8.0), 170$ $\mathrm{mM} \mathrm{KCl}, 7.5 \mathrm{mM} \mathrm{MgCl} 2,0.1 \mathrm{mM}$ EDTA, $1 \mathrm{mM}$ DTT, and $1 \%$ Triton X-100] for $3 \mathrm{~h}$ on a rotator at $4^{\circ} \mathrm{C}$. After being 


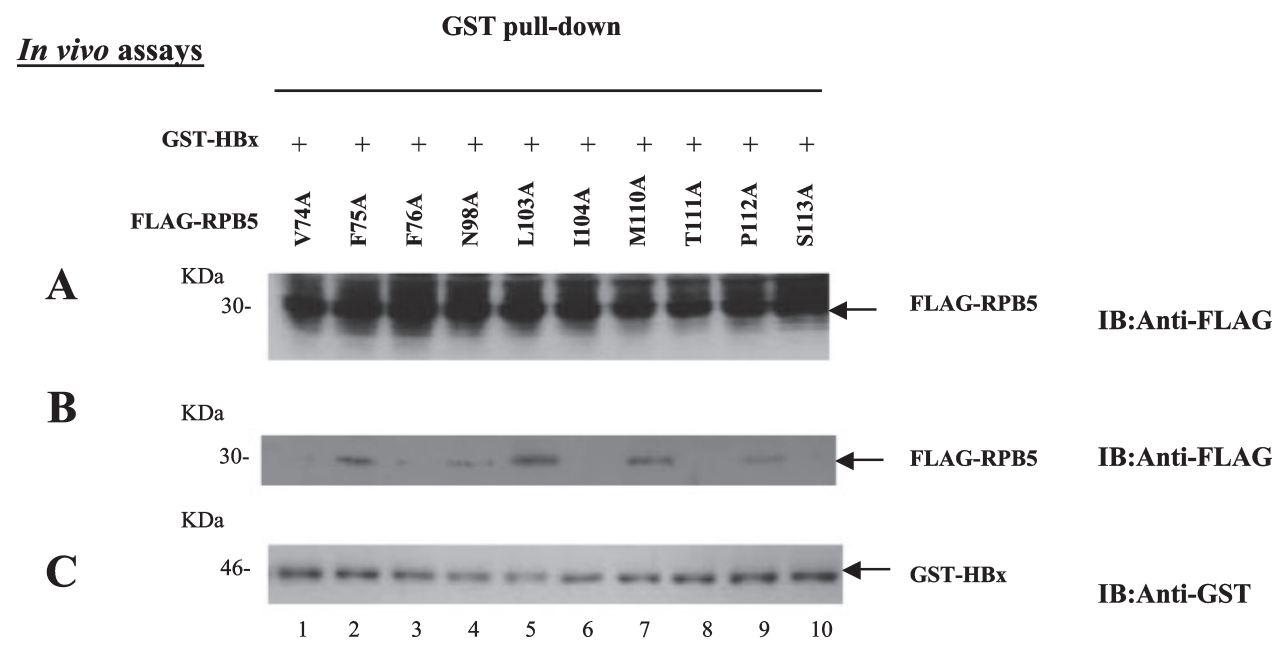

$\underline{\text { In vitro assays }}$

D

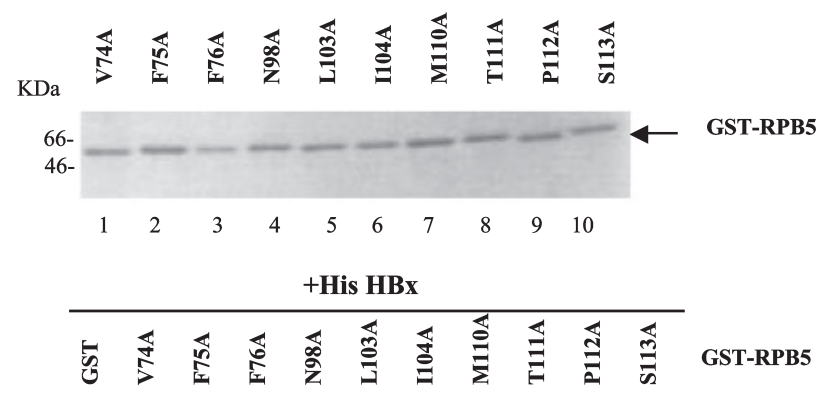

$\mathbf{E}$

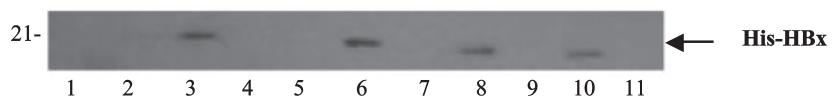

\section{IB:Anti-His}

Fig. 3. Amino acid residues of RPB5 critical for the HBxbinding as analyzed in vivo and in vitro. The experiments were carried out as in Fig. 2 except that pNKFLAG-RPB5 harboring a single amino acid substitution (pm) as indicated at the top was used. (A-C) In vivo assays: (A) Western blot analysis of total lysates with an $\alpha$ FLAG monoclonal antibody. (B) Western blot analysis of recovered proteins bound to glutathione resin with the $\alpha$-FLAG monoclonal antibody. (C) The nitrocellulose membranes used for Western blot analysis with the $\alpha$-FLAG antibody were reprobed with an $\alpha$-GST antibody. (D and E) In vitro assays: (D) Purified bacterially expressed GST-RPB5 pm proteins were separated by SDS-PAGE and stained with Coomassie Brilliant Blue. (E) Recovered HisHBx protein bound to GST or GST-RPB5 pm protein detected with an $\alpha$-His monoclonal antibody. Each experiment was repeated at least three times and the data were reproducible. washed four times with GBT buffer, the bound proteins were eluted, fractionated by $12 \%$ SDS-PAGE, and subjected to Western blot analysis using anti-FLAG monoclonal antibody (M2) or anti-His monoclonal antibody.

Preparation of Cell Extract, Coprecipitation with Glutathione-Sepharose $4 B$ Resin, and Western Blot Analysis - The transient transfection of COS1 cells was carried out by standard $\mathrm{CaCl}_{2}$-mediated transfection. The cells were harvested, washed, and sonicated in lysis buffer [10\% glycerol, $20 \mathrm{mM}$ HEPES ( $\mathrm{pH} 7.9$ ), $50 \mathrm{mM}$ $\mathrm{KCl}, 0.4 \mathrm{M} \mathrm{NaCl}, 1 \mathrm{mM} \mathrm{MgCl}{ }_{2}, 0.1 \mathrm{mM}$ dithiothreitol, 0.1 mM EDTA, 9 mM CHAPS, $0.5 \mathrm{mM}$ phenylmethylsulfonyl fluoride, and $10 \mu \mathrm{g} / \mathrm{ml}$ aprotinin and leupeptin], and then centrifuged. The total cell lysates were stored at $-80^{\circ} \mathrm{C}$. Total cell lysates were diluted with 4 times the volume of buffer [50 mM Tris-HCl (pH 7.5), $150 \mathrm{mM} \mathrm{NaCl,} \mathrm{and}$ $0.05 \%$ Tween 20] and incubated with $50 \mu \mathrm{l}$ of glutathioneSepharose $4 \mathrm{~B}$ beads for $3 \mathrm{~h}$ on a rotator at $4^{\circ} \mathrm{C}$. After extensive washing, the bound proteins were eluted, fractionated by $15 \%$ SDS-PAGE, transferred onto nitrocellulose membranes, and subjected to Western blot analysis with the anti-FLAG M2 antibody. The proteins were visualized by enhanced chemiluminescence (ECL) according to the manufacturer's instructions (Amersham Biosciences). The nitrocellulose membranes used for Western blot analysis with anti-FLAG monoclonal anti- body were reprobed with anti-GST monoclonal antibody (Zymed Laboratories Inc.) according to the manufacturer's instructions (Amersham Biosciences).

Cell Culture and Transient Transfection-COS1 cells (a monkey kidney cell line) were cultured in Dulbecco's Modified Eagle's Medium (DMEM; Invitrogene Co) supplemented with $5 \%$ fetal calf serum (FCS; CELLect R GOLD) and $20 \mathrm{mg} / \mathrm{ml}$ antibiotics (ampicillin and kanamycin; Meigi Co. Ltd ) and maintained in an incubator with $5 \% \mathrm{CO}_{2}$ at $37^{\circ} \mathrm{C}$.

\section{RESULTS}

Amino Acid Sequences of the Central Part of RPB5 Necessary for HBx-Binding In Vivo and In Vitro-The HBxbinding region has been mapped within the central part of RPB5 using various versions of truncation mutants (15). To specify the amino acid sequence(s) and amino acid residue(s) critical for $\mathrm{HBx}$-binding, we applied a twostep alanine scanning method using clustered substitution mutants $(\mathrm{cm})$ and point substitution mutants $(\mathrm{pm})$. A cm library of human RPB5 covering aa 21 to 132 was constructed by systematically substituting 7 amino acid residues in a row with AAASAAA as shown in Fig. 1.

The ability of these cm RPB5 proteins to bind HBx was examined with lysates of mammalian cells transiently 
Table 1. Summary of RPB5-binding abilities.

\begin{tabular}{cccccc}
\hline $\begin{array}{c}\text { Mutated } \\
\text { human RPB5 residue }\end{array}$ & HBx & RAP30 (TFIIF) & $\begin{array}{c}\text { Mutated } \\
\text { human RPB5 residue }\end{array}$ & HBx & RAP30 (TFIIF) \\
\hline T56A & $+^{\mathrm{a}}$ & - & I99A & ++ & ++ \\
D57A & ++ & ++ & T100A & ++ & ++ \\
L58A & ++ & - & R101A & ++ & ++ \\
D70A & ++ & ++ & L103A & ++ & ++ \\
Q71A & ++ & ++ & I104A & - & - \\
M72A & ++ & ++ & V105A & ++ & ++ \\
F73A & ++ & ++ & M110A & ++ & ++ \\
V74A & - & ++ & T111A & - & - \\
F75A & ++ & ++ & P112A & ++ & ++ \\
F76A & - & - & S113A & - & - \\
N98A & - & ++ & & & \\
\hline
\end{tabular}

a(++): binding positive; (-): binding negative.

coexpressing GST-HBx and each FLAG-RPB5 $\mathrm{cm}$ protein in vivo. COS1 cells were transfected with the mammalian expression vectors and the cell lysates were pull-downed with glutathione resin. GST-HBx recovered FLAG-RPB5 while GST alone did not (Fig. 2B, lanes 2 and 3). The specific interaction of two proteins was also detected with a different combination of tagged proteins (data not shown). Under these conditions, four $\mathrm{cm}$ proteins of RPB5 (cm 70, cm 98, cm 105, and cm 112) were not recovered with $\mathrm{HBx}$ (Fig. 2B lanes 4, 8, 9, and 10, respectively), although the other three $\mathrm{cm}$ proteins were recovered similar to the wild type RPB5, and $\mathrm{cm} 91$ was recovered, but inefficiently (Fig. 2B, compared lane 3, and 7 with lanes 5,6 , and 11 , respectively). This result was not due to different expression levels of proteins and/ or the efficiency of GST pull-down since the recovery of GST-fused proteins was similar (Fig. 2C) and the expression levels of FLAG-RPB5 wild type (WT) and clustered mutants were similar (Fig. 2A). Each experiment was repeated at least three times and the data are reproducible.

To confirm the binding properties of $\mathrm{cm}$ mutants to HBx in vivo, we performed an in vitro pull-down assay. Bacterially expressed wild type and cm GST-RPB5 were bound to glutathione resin (Fig. 2D) and subjected to binding assays with purified His-tagged $\mathrm{HBx}$ (as described in "MATERIALS AND METHODS"). To purify the $\mathrm{HBx}$ protein expressed in vitro, the denatured followed by renaturation method is better than the native method (see details of purification in "MATERIALS AND METHODS"). The bound HBx was subjected to Western blot analysis with anti-His antibody. Consistent with the result in vivo, we observed that the four cm RPB5 (cm 70, $\mathrm{cm} \mathrm{98,}$ $\mathrm{cm} \mathrm{105,} \mathrm{and} \mathrm{cm} \mathrm{112)} \mathrm{were} \mathrm{unable} \mathrm{to} \mathrm{interact} \mathrm{with} \mathrm{HBx}$ (Fig. 2E lanes 4, 8, 9, and 10, respectively). The other $\mathrm{cm}$ mutants retained the ability to interact with $\mathrm{HBx}$, although the amount of protein recovered was not the same for each mutant (Fig. 2E lanes 5, 6, 7, 11 and data not shown). Four sequences of RPB5 (cm70, cm98, cm105 and $\mathrm{cm} 112$ ) critical for $\mathrm{HBx}$-binding in vivo and in vitro are in two different areas within the central part of RPB5 (aa 53 to 136).

Interaction between $H B x$ and Alanine Point Mutant (pm) RPB5 In Vivo and In Vitro-To identify residues critical for binding $\mathrm{HBx}$ within the four cm mutants, we constructed a second series of $\mathrm{cm}$ mutants harboring
AAASA and SAAA in the target sequence using the NheI site-encoding SA sequence and analyzed them for protein-protein interactions (data not shown). Then single alanine substitution mutants covering the sequence(s) were constructed as explained in "MATERIALS AND METHODS." Similar experiments with the pm mutant series of RPB5 were conducted in vivo and in vitro. We failed to detect any interaction of HBx with RPB5 having V74A, I104A, T111A, or S113A in both the in vivo and in vitro assays (Fig. 3B, lanes 1, 6, 8, and 10, respectively; Fig. $3 \mathrm{E}$, lanes $2,7,9$, and 11 , respectively). RPB5 harboring F76A or N98A are severely affected in vivo and completely defective in vitro in their ability to interact with HBx (Fig. 3B, lanes 3 and 4, respectively; Fig. 3E, lanes 4 and 5 , respectively). On the other hand, none of the other residues listed in Table 1 impaired the $\mathrm{HBx}$ interaction (Fig. 3B, lanes 2, 5, 7, and 9; Fig. 3E, lanes 3, 6, 8, and 10; and data not shown). Taken together, the results of the experiments with coexpressed proteins in COS1 cells are consistent with those with purified bacterial proteins in vitro, indicating that the RPB5 residues critical for $\mathrm{HBx}$ interaction are V74, F76, N98, I104, T111, and S113.

Interaction of RPB5 and (RAP30) TFIIF Proteins In Vivo and In Vitro Analyzed with $\mathrm{cm}$ and pm Mutants of RPB5-Previously we reported a direct interaction between RPB5 and subunit RAP30 of TFIIF (18); however, the residues within RPB5 responsible for this interaction remain unknown. In this study, we addressed the question of which sequences in the central part of RPB5 are required for the interaction between RAP30 in the TFIIF complex and RPB5. To this end, COS1 cells were cotransfected with mammalian plasmids expressing two subunits of TFIIF: GST-RAP30 and FLAG-RAP74 together with wild type or one of the cm mutants of FLAG-RPB5. The cell lysates were subjected to GST pulldown with glutathione resin, and the recovered FLAGtagged RPB5 and RAP74 were detected immunologically using an anti-FLAG monoclonal antibody. All the clustered mutants of RPB5 were expressed equally upon transfection into COS1 cells, and GST-RAP30 in each combination of cotransfection was similarly recovered by GST pull-down (Fig. 4, A and C). GST-RAP30, but not GST alone, recovered FLAG-RPB5 and FLAG-RAP74 (Fig. 4B, lanes 2 and 3). The five $\mathrm{cm}$ mutants $(\mathrm{cm} 56, \mathrm{~cm}$ $70, \mathrm{~cm} \mathrm{98,} \mathrm{cm} \mathrm{105,} \mathrm{and} \mathrm{cm} \mathrm{112)} \mathrm{were} \mathrm{impaired} \mathrm{in} \mathrm{their}$ interaction with RAP30 in the TFIIF complex (Fig. 4B, 


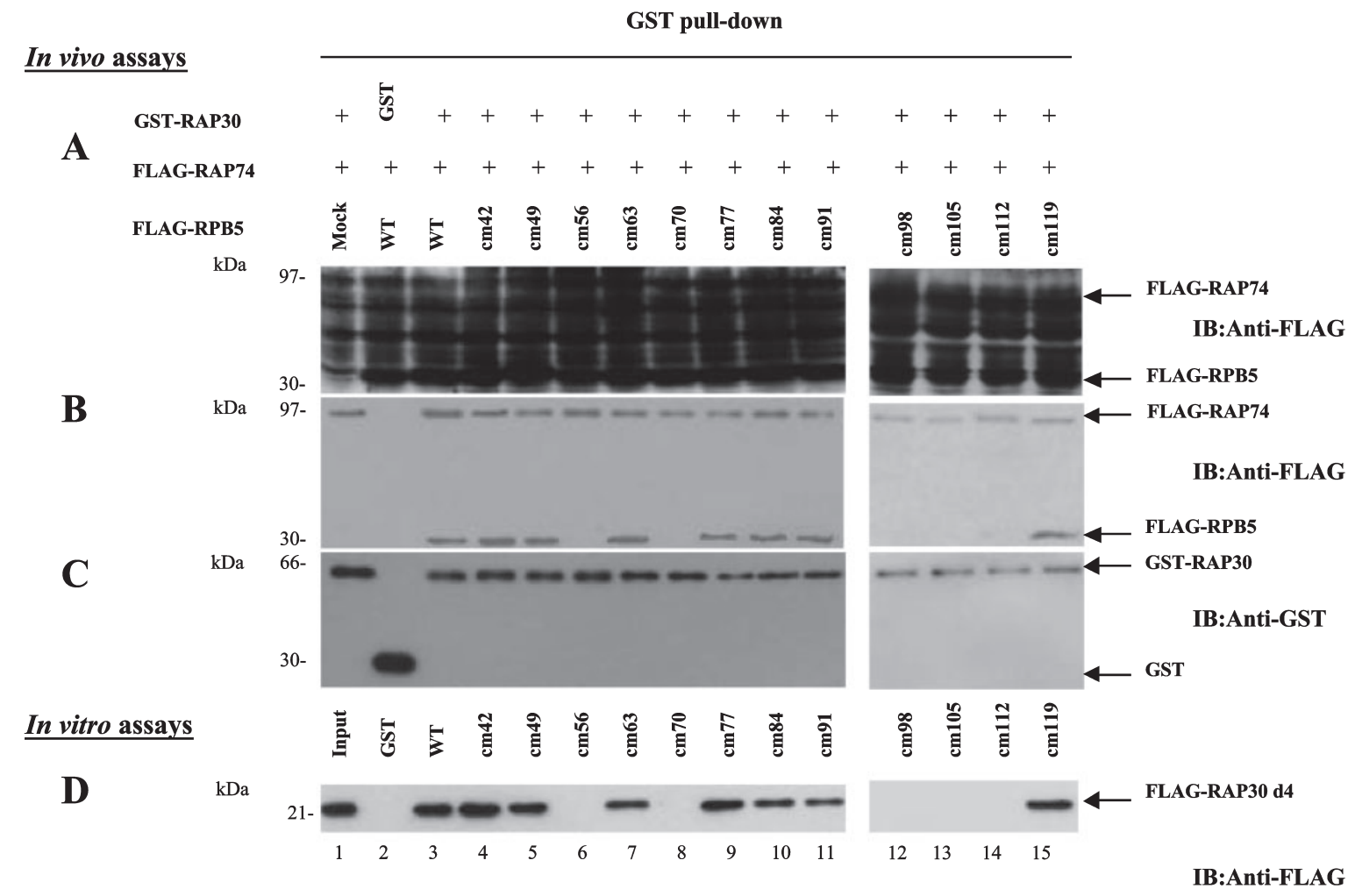

Fig. 4. Amino acid sequences of RPB5 critical for RAP30-binding in the TFIIF complex as analyzed in vivo and in vitro. (AC) In vivo assays: Experiments were conducted in COS1 cells as described in Fig. 2, A-C, except that COS1 was transiently cotransfected with the mammalian expression vectors pNKGST-RAP30 and pNKFLAG-RAP74 in the absence of pNKFLAG-RPB5 (mock) (lane

lanes 6, 8, 12, 13 and 14, respectively), although similar amounts of RAP74 were recovered in the presence or absence of wild type or mutant FLAG-RPB5. In contrast, the other clustered mutants of RPB5 did show an ability to bind TFIIF similar to that of the wild type RPB5 (Fig. $4 \mathrm{~B}$, lanes $3,4,5,7,9,10,11,15$ and data not shown). Interestingly, all five of these $\mathrm{cm}$ mutants, except $\mathrm{cm} 56$, are not only defective in TFIIF-binding but also $\mathrm{HBx}$ binding (Fig. 2), indicating that overlapping sequences are involved in the bindings to the two factors.

As previously reported, the N-terminus and the middle part of subunit RAP30 can interact independently with RPB5, although the former is masked by RAP74 in the TFIIF complex (18). Therefore, the middle part of RAP30, the construct RAP30 d4 (aa 101 to 249), was tested for binding. It could bind RPB5 regardless of the presence or absence of RAP74, a binding consistent with a previous report (data not shown). Based on this observation, next we used RAP30 d4 (aa 101 to 249) for the pull-down assay in vitro to evaluate its ability to bind bacterially expressed and purified wild type or cm RPB5. As shown in Fig. 4D, all proteins except $\mathrm{cm} 56, \mathrm{~cm} \mathrm{70,} \mathrm{cm} \mathrm{98,} \mathrm{cm}$ 105 , and $\mathrm{cm} \mathrm{112,} \mathrm{were} \mathrm{recovered} \mathrm{with} \mathrm{GST-RPB5}$ bounded resin. Although we observed that $\mathrm{cm} 63, \mathrm{~cm} 84$, cm 91 showed weaker binding ability (Fig. 4D, compared lanes 3, 4, 5, 9 and 15 with lanes 7, 10, and 11).

Using the same method as described in "MATERIALS AND METHODS" to pinpoint critical residues of RPB5 for
1), or together with pNKFLAG-RPB5 WT or $\mathrm{cm}$ as indicated. (D) In vitro assays: Recovered bacterially expressed FLAG-RAP30 d4 (see text in detail) bound to GST or GST-RPB5 WT or cm protein prebound to glutathione resin was detected by Western blot analysis with an $\alpha$-FLAG antibody. Each experiment was repeated at least three times and the data were reproducible.

RAP30-binding, we further constructed mutants of RPB5 with point alanine substitutions and examined their ability to interact with RAP30 (subunit of TFIIF) both in vivo and in vitro. The six pm mutants of RPB5 (T56A, L58A, F76A, I104A, T111A, and S113A) were clearly defective in both RAP30-binding in the TFIIF complex in vivo and in RAP30-binding using RAP30 d4 (which binds RPB5 regardless of the presence or absence of RAP74) in vitro (Fig. 5B, lanes 1, 3, 5, 7, 9, and 11, respectively; Fig. 5D, lanes $2,4,6,8,10$, and 12 , respectively). In contrast, other pm mutants of RPB5 listed in Table 1 were able to bind (Fig. 5B, lanes 2, 4, 6, 8 and 10; Fig. 5D, lanes 3, 5, 7, 9 , and 11; and data not shown). Interestingly, among them, in the in vivo experiment, two pm mutants, D57A and P112A, exhibited much weaker ability to bind RAP30; however, in vitro, pm mutant L103A exhibited weaker interaction, suggesting that the GST pull-down assay with two purified proteins in vitro may be not the same as the GST pull-down of total cell lysate proteins in vivo.

Position of Residues Critical for the Interaction of Human RPB5 with HBx/RAP30-Collectively, in the central part of human RPB5, we identified six residues critical for the interaction with $\mathrm{HBx}$ and six residues indispensable for the interaction with RAP30. Interestingly, among them, four residues (hF76, hI104, hT111, and hS113) are required for the interactions with both HBx and RAP30 in the TFIIF complex, and are conserved the among human and yeast proteins (Table 1). The 


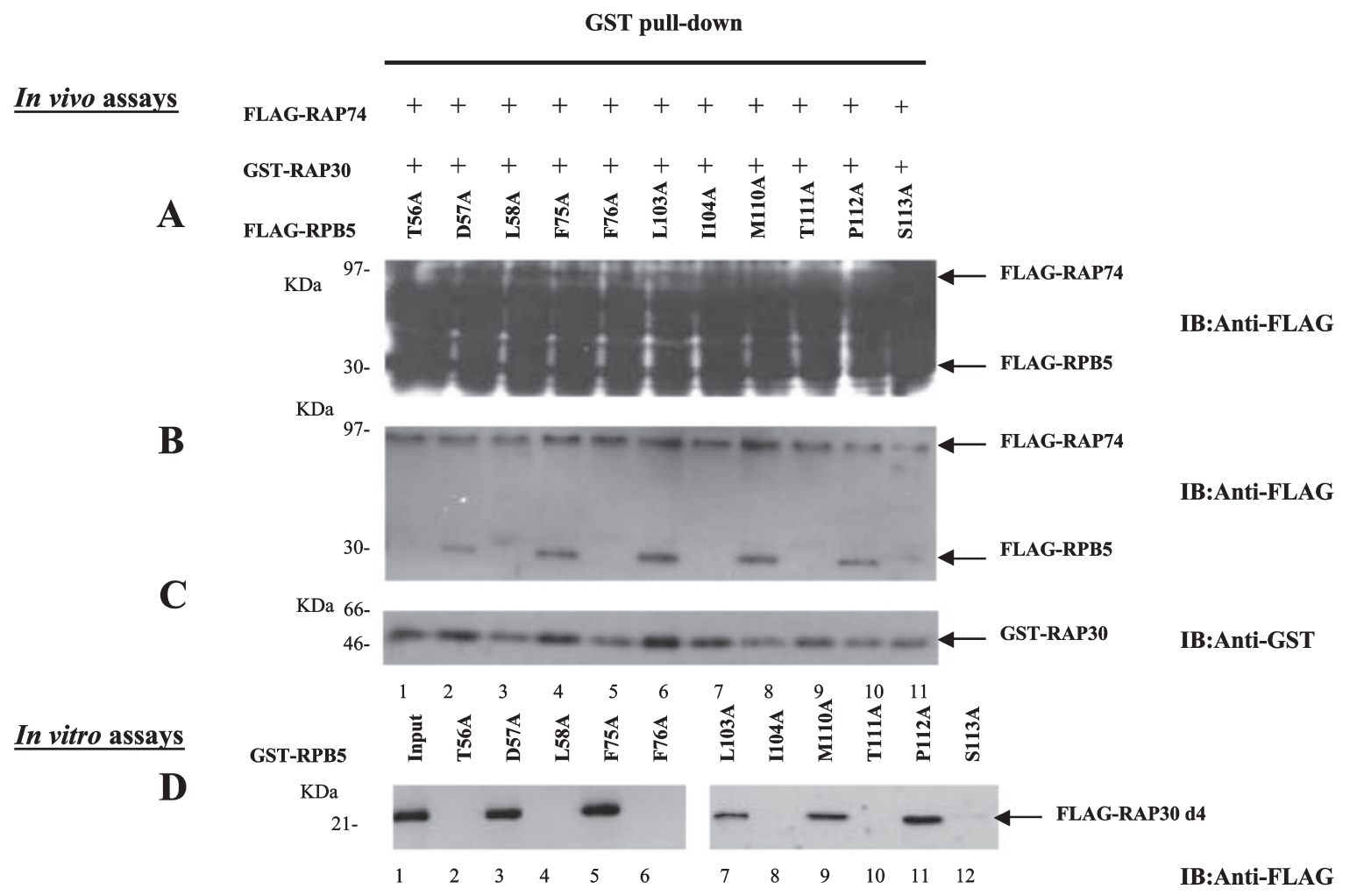

Fig. 5. Amino acid residues of RPB5 critical for the RAP30- RPB5 proteins with a single amino acid substitution as indicated binding in the TFIIF complex as analyzed in vivo and in vitro. (A-C) In vivo assays: The experiments were conducted in COS1 cells as described in Fig. 4 except that pNKFLAG-RPB5 pm as indicated at the top was used. (D) In vitro assays: bacterially expressed GST-

newly identified critical amino acid residues of hRPB5 and the corresponding conserved residues in yRPB5 are shown in Fig. 6. Among the four conserved residues, yF82 (hF76) is juxtaposed in a $\beta$-sheet [designated A2 (21)] in parallel with a $\beta$-sheet (A3) harboring yI109 (hI104), which is close to yF82 (hF76). Amino acids yT117 (hT111) and yS119 (hS113) are at the top of the exposed helix or in the loop region (Fig. 6), and are the nearest neighbors of yP118 (hP112), which has been proposed to be one of the residues closest to the DNA. The two residues specific for RAP30-binding, hT56 and hL58, are in the loop between the N-terminal part and the central part of RPB5, and are not conserved among human and yeast. The two residues specific for $\mathrm{HBx}$ interaction, yV80 (hV74) and yN104 (hN98), are in $\mathrm{A} 3$ and in a loop between a helix $(\alpha 5)$ and $\mathrm{A} 3$.

\section{DISCUSSION}

Here, we report six residues (V74, F76, N98, I104, T111 and S113) critical for HBx-binding, and six residues (T56, L58, F76, I104, T111 and S113) critical for RAP30-binding by RPB5. Interestingly, four of these residues are redundant for RAP30- and HBx-binding, strongly implying that the two factors compete with each other for RPB5-binding. In this context, HBx may inhibit or delay a step in transcription in which the direct interaction between RPB5 and TFIIF is involved. TFIIF has several contact sites for RNAPII during initiation and elongation

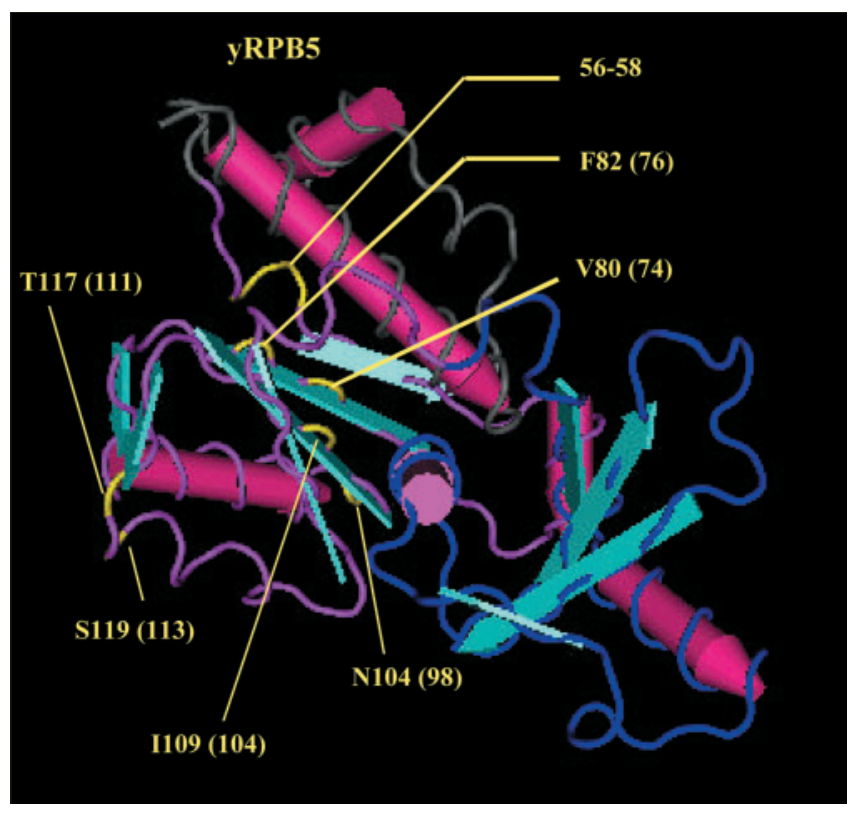

Fig. 6. Position of the newly identified residues in the crystal model of RPB5. The corresponding conserved amino acid residues predicted in Saccharomyces cerevisie RPB5 are shown in yellow (except residues 56 to 58 belonging to a non-conserved region), and the amino acid residues of human RPB5 essential for binding HBx and the RAP30 subunit of TFIIF: threonine 56 (T56), leucine 58 (L58), valine 74 (V74), phenylalanine 76 (F76), asparagine 98 (N98), isoleucine 104 (I104), threonine 111 (T111), and serine 113 (S113), are shown in parentheses. 
of transcription, and the initiation step before the interaction of RPB5 and TFIIF may be critical for activated transcription. Another possibility is that the central region of the exposed domain covering aa 72 to 120 may be an important surface for unidentified factors (as discussed below).

The central part of RPB5 (aa 53 to 136), proximal to DNA downstream of the promoter (see Fig. 6), acts in communication with several transcriptional regulators, the TFIIF subunit RAP30, HBx and a novel corepressor RMP/URI $(8,13,15,16,18,20,21,36,38)$. We previously identified $\mathrm{HBx}$ as a transcriptional coactivator through its direct interaction with the central part of RPB5 (15, $17,33,34)$. The putative role of human RPB5 in activated transcription is consistent with the reported role of yeast RPB5 in the activated transcription of some promoters (38), although it remains uncertain how RPB5 is involved in activated transcription but not in basal transcription. By two-step scanning with clustered and point alanine substitution(s) libraries, we dissected the central part of hRPB5 to address whether the sequence and residues needed for RAP30-binding and HBx-binding are redundant. In this paper, the interaction of RPB5 with HBx and TFIIF was examined in vitro and in vivo with RPB5 and its partner co-expressed transiently in cells. The interaction of RPB5 in RNAPII with these factors could be elucidated since ectopically expressed RPB5 can be efficiently incorporated into RNAPII (data not shown).

As RPB5 is well conserved between yeast and human $(8,10,13,15,18,21,22,38,39)$, and the topology of the preinitiation complex is remarkably similar in the two species as analyzed by cross-link experiments and cryoelectron microscopy $(11,12,32)$, the relevance of the positions of the conserved residues in human RPB5 were evaluated on basis of the yeast crystal model (Fig. 6). The results suggest that the rigid property of the fourstranded $\beta$-sheet may be important for the structural integrity of the central part of RPB5 (aa 53 to 144 of yRPB5 or aa 53 to 139 of hRPB5). All residues except T117 and S119 are non-charged and hydrophobic in the four-stranded mixed $\beta$-sheet. As all the RPB5 residues critical for binding RAP30 and/or HBx are conserved between yeast and human except aa 56 to 58, we next addressed the biological role of the six conserved residues in yRPB5 by introducing point mutations in yeast in place of the wild type counterpart. The yeast rpb5 series of alanine/glycine substituted point mutant alleles was generated by two-step PCR mutagenesis. The mutations had no influence on cell viability but affected yeast cell growth, especially at suboptimal temperatures (ts or/and cs phenotype) (unpublished data). These results clearly support the notion that the conserved residues of RPB5 required for RAP30-binding as well as $\mathrm{HBx}$-binding play an important role in cell growth in yeast. Interestingly, the two charged residues, T117 and S119 of yRPB5 (T111 and S113 of hRPB5), are exposed to the solvent, and are the nearest neighbors of the proline (yP118) that has been reported to be closest to the DNA (8). We identified the DNA-binding properties of hRPB5, and showed that T111 and S113, but not P112, are critical or important for DNA-binding (Zhang S. and Murakami S. et al, in a separate manuscript). DNA has been reported to wrap around the yeast and human RNAPII complex $(9,29,30,32)$, and TFIIF forms multiple DNA contacts, spanning the DNA from upstream to downstream of the promoter as detected in cross-linking experiments $(11,23,30,32)$. In that context, it is possible that both TFIIF and HBx may modulate the interactions of RNAPII and DNA by preventing RPB5 from accessing DNA downstream of the initiation site. In this respect, it remains to be addressed whether RPB5 is involved in recognition of a novel downstream promoter element, MTE (motif ten element), that is conserved from Drosophila to human, as recently reported (40).

We further defined the sequences of $\mathrm{HBx}$ that are important for RPB5-binding (Le T. and Murakami S. et $a l$. , unpublished data). All sequences are in the coactivation domain of $\mathrm{HBx}$, but, interestingly, they are much narrower than those necessary for the coactivation function of $\mathrm{HBx}$ as analyzed with the clustered mutant library of $\mathrm{HBx}$ (35). The results imply that multiple partners are necessary for the coactivation or transactivation function of $\mathrm{HBx}$, in addition to RPB5 as reviewed (17).

$\mathrm{RMP} / \mathrm{URI}(16,36)$ is another factor that requires the central part of RPB5 for interaction. Three out of the four residues critical for the binding of both RAP30 and $\mathrm{HBx}$ seem also to be indispensable for the binding of RMP/URI (in preparation). Gstaiger, et al. clearly demonstrated that RMP/URI belongs to a prefoldin (PFD) family whose members are able to assemble into molecular chaperone complexes (36), and that yeast and human RMP/URIs both seem to be targets of nutrient signaling controlled by mTOR kinase in the cytoplasm $(19,36)$. Therefore, one possibility is that the role of RPB5 in activated transcription is not due to the function of RPB5 assembled in RNAPII, and that HBx may induce the dissociation of the cytoplasmic RPB5 and RMP/URI complex and facilitate the assembly of nuclear RPB5 in RNAPII as HBx can inhibit the binding between RPB5 and RMP/URI (unpublished data). Recently, we reported the nuclear role of RMP/URI in a complex with DMAP1, DNA methyltransferase 1-interacting protein, and showed that this complex exhibits corepressor activity (19). In this context, it seems possible that $\mathrm{HBx}$ releases the corepressor complex RMP and DMAP1, and facilitates activated transcription. The role of RPB5 in activated transcription remains to be elucidated.

Taken together, the central part of RPB5 proximal to DNA downstream of the promoter can be a target of several transcriptional regulators that may interfere with the interaction between the exposed domain of RPB5 and DNA.

We thank Dr. Tang (Sichuan University, China) for valuable discussions. We thank Dr. Hirose (Kanazawa University) for kind help to improve our manuscript. We thank Ms. Kuwabara for excellent technical assistance. This work was supported in part by a Grant-in-aid for Scientific Research (B) and Development, a Grant-in-Aid for Scientific Research on Priority Areas (C) from the Ministry of Education, Culture, Sports, and Technology, and Grant-in-Aid for Cancer Research from the Ministry of Health, Labor, and Welfare of Japan. 


\section{REFERENCES}

1. Roeder, R.G. (1996) The role of general initiation factors in transcription by RNA polymerase II. Trends Biochem. Sci. 21, $327-335$

2. Hampsey, M. (1998) Molecular genetics of the RNA polymerase II general transcriptional machinery. Microbiol. Rev. 62, 465-503

3. Cramer, P., Bushnell, D.A., and Kornberg, R.D. (2001) Structural basis of transcription: RNA polymerase II at 2.8 angstrom resolution. Science 292, 1863-1876

4. Gnatt, A.L., Cramer, P., Fu, J., Bushnell, D.A., and Kornberg, R.D. (2001) Structural basis of transcription: an RNA polymerase II elongation complex at $3.3 \AA$ resolution. Science 292, $1876-1882$

5. Hahn, S. (2004) Structure and mechanism of the RNA polymerase II transcription machinery. Nat. Struct. Mol. Biol. 11, 394-403

6. Rani, P.G., Ranish, J.A., and Hahn, S. (2004) RNA polymerase II (Pol II)-TFIIF and Pol II-mediator complexes: the major stable Pol II complexes and their activity in transcription initiation and reinitiation. Mol. Cell. Biol. 24, 1709-1720

7. Bushnell, D.A., Westover, K.D., Davis, R.E., and Kornberg, R.D. (2004) Structural basis of transcription: an RNA polymerase II-TFIIB cocrystal at 4.5 Angstroms. Science 303, 983-988

8. Cramer, P., Bushnell, D.A., Fu, J., Gnatt, A.L., Maier-Davis, B., Thompson, N.E., Burgess, R.R., Edwards, A.M., David, P.R., and Kornberg, R.D. (2000) Architecture of RNA polymerase II and implications for the transcription mechanism. Science 288, 640-649

9. Woychik, N.A. and Hampsey, M. (2002) The RNA polymerase II machinery: structure illuminates function. Cell 108, 453-463

10. Bushnell, D.A. and Kornberg, R.D. (2003) Complete, 12-subunit RNA polymerase II at 4.1-Å resolution: implications for the initiation of transcription. Proc. Natl Acad. Sci. USA 100, 6969-6973

11. Chung, W.-H., Craighead, J.L., Chang, W.-H., Ezeokonkwo, C., Bareket-Samish, A., Kornberg, R.D., and Asturias, F.J. (2003) RNA polymerase II/TFIIF structure and conserved organization of the initiation complex. Mol. Cell 12, 1003-1013

12. Chen, B.S., Mandal, S.S., and Hampsey, M. (2004) High-resolution protein-DNA contacts for the yeast RNA polymerase II general transcription machinery. Biochemistry 43, 1274112749

13. Cheong, J.H., Yi, M., Lin, Y., and Murakami, S. (1995) Human RPB5, a subunit shared by eukaryotic nuclear RNA polymerases, binds human hepatitis $\mathrm{B}$ virus $\mathrm{X}$ protein and may play a role in X transactivation. EMBO J. 14, 143-150

14. Cho, H., Maldonado, E., and Reinberg, D. (1997) Affinity purification of a human RNA polymerase II complex using monoclonal antibodies against transcription factor IIF. J. Biol. Chem. 272, 11495-11502

15. Lin, Y., Nomura, T., Cheong, J.H., Dorjsuren, D., Iida, K., and Murakami, S. (1997) Hepatitis B virus X protein is a transcriptional modulator that communicates with transcriptional factor IIB and RNA polymerase II subunit 5. J. Biol. Chem. 272, $7132-7139$

16. Dorjsuren, D., Lin, Y., Wei, W., Yamashita, T., Nomura, T., Hayashi, N., and Murakami, S. (1998) RMP, a novel RNA polymerase II subunit 5 -interacting protein, counteracts transactivation by hepatitis B virus X protein. Mol. Cell. Biol. 18, $7546-7555$

17. Murakami, S. (2001) Hepatitis B virus X protein: multifunctional viral regulator. J. Gastroenterol. 36, 651-660

18. Wei, W., Dorjsuren, D., Lin, Y., Qin, W., Nomura, T., Hayashi, N., and Murakami, S. (2001) Direct interaction between the subunit RAP30 of transcription factor IIF (TFIIF) and RNA polymerase subunit 5 , which contributes to the association between TFIIF and RNA polymerase II. J. Biol. Chem. 276, 12266-12273

19. Luvsanjav, D., Hayashi, N., Dorjsuren, D., Nomura, T., Le, T.T.T., and Murakami, S. (2004) Subcellular localization of RPB5- mediating protein and its putative functional partner. Mol. Cell. Biol. 24, 8556-8566

20. Hodach, M., Todone, F., Eloranta, J.J., Onesti, S., and Weinzierl, R.O. (1999) Crystallization and preliminary diffraction studies of the RNA polymerase subunit RPB5 from Saccharomyces cerevisiae. Acta. Crystallogr. D Biol. Crystallogr. 55, 1373-1375

21. Todone, F., Weinzierl, R.O., Brick, P., and Onesti, S. (2000) Crystal structure of RPB5, a universal eukaryotic RNA polymerase subunit and transcription factor interaction target. Proc. Natl Acad. Sci. USA 97, 6306-6310

22. Yee, A., Booth, V., Dharamsi, A., Engel, A., Edwards, A.M., and Arrowsmith, C.H. (2000) Solution structure of the RNA polymerase subunit RPB5 from Methanobacterium thermoautotrophicum. Proc. Natl Acad. Sci. USA 97, 6311-6315

23. Tan, S., Conaway, R.C., and Conaway, J.W. (1995) Dissection of transcription factor TFIIF functional domains required for initiation and elongation. Proc. Natl Acad. Sci. USA 92, 60426046

24. Orphanides, G., Lagrange, T., and Reinberg, D. (1996) The general transcription factors of RNA polymerase II. Genes Dev. 10, 2657-2683

25. Sopta, M., Carthew, R.W., and Greenblatt, J. (1985) Isolation of three proteins that bind to mammalian RNA polymerase II. $J$. Biol. Chem. 260, 10353-10360

26. Flores, O., Lu, H., Killeen, M., Greenblatt, J., Burton, Z.F., and Reinberg, D. (1991) The small subunit of transcription factor IIF recruits RNA polymerase II into the preinitiation complex. Proc. Natl Acad. Sci. USA 88, 9999-10003

27. Killeen, M.T. and Greenblatt, J.F. (1992) The general transcription factor RAP30 binds to RNA polymerase II and prevents it from binding nonspecifically to DNA. Mol. Cell. Biol. 12, 30-37

28. Jeronimo, C., Langelier, M.-F., Zeghouf, M., Cojocaru, M., Bergeron, D., Baali, D., Forget, D., Mnaimneh, S., Davierwala, A.P., Pootoolal, J., Chandy, M., Canadien, V., Beattie, B.K., Richards, D.P., Workman, J.L., Hughes, T.R., Greenblatt, J., and Coulombe, B. (2004) RPAP1, a novel human RNA polymerase II-associated protein affinity purified with recombinant wild-type and mutated polymerase subunits. Mol. Cell. Biol. 24, 7043-7058

29. Kim, T.K., Lagrange, T., Wang, Y.H., Griffith, J.D., Reinberg, D., and Ebright, R.H. (1997) Trajectory of DNA in the RNA polymerase II transcription preinitiation complex. Proc. Natl Acad. Sci. USA 94, 12268-12273

30. Robert, F., Douziech, M., Forget, D., Egly, J.M., Greenblatt, J., Burton, Z.F., and Coulombe, B. (1998) Wrapping of promoter DNA around the RNA polymerase II initiation complex induced by TFIIF. Mol. Cell 2, 341-351

31. Langelier, M.F., Forget, D., Rojas, A., Porlier, Y., Burton, Z.F., and Coulombe, B. (2001) Structural and functional interactions of transcription factor (TF) IIA with TFIIE and TFIIF in transcription initiation by RNA polymerase II. J. Biol. Chem. 276, 38652-38657

32. Forget, D., Langelier, M.F., Therien, C., Trinh, V., and Coulombe, B. (2004) Photo-cross-linking of a purified preinitiation complex reveals central roles for the RNA polymerase II mobile clamp and TFIIE in initiation mechanisms. Mol. Cell. Biol. 24, 1122-1131

33. Lin, Y., Tang, H., Nomura, T., Dorjsuren, D., Hayashi, N., Wei, W., Ohta, T., Roeder, R., and Murakami, S. (1998) The hepatitis $\mathrm{B}$ virus $\mathrm{X}$ protein is a co-activator of activated transcription that modulates the transcription machinery and distal binding activators. J. Biol. Chem. 273, 27097-27103

34. Murakami, S. (1999) Hepatitis B virus X protein: structure, function and biology. Intervirology 42, 81-99

35. Tang, H., Luvsanjav, D., Feijun, H., Naoki, O., Li, L., Fang, H., Liansan, Z., and Murakami, S. (2005) Transcriptional transactivation function of $\mathrm{HBx}$ protein is important for its augmentation role in hepatitis B virus replication. J. Virol. in press

36. Gstaiger, M., Luke, B., Hess, D., Oakeley, E.J., Wirbelauer, C., Blondel, M., Vigneron, M., Peter, M., and Krek, W. (2003) Con- 
trol of nutrient-sensitive transcription programs by the unconventional prefoldin URI. Science 302, 1208-1212

37. Tang, H., Sun, X., Reinberg, D., and Ebright, R.H. (1996) Protein-protein interactions in eukaryotic transcription initiation: structure of the preinitiation complex. Proc. Natl Acad. Sci. USA 93, 1119-1124

38. Miyao, T. and Woychik, N.A. (1998) RNA polymerase subunit RPB5 plays a role in transcriptional activation. Proc. Natl Acad. Sci. USA 95, 15281-15286
39. McKune, K., Moore, P.A., Hull, M.W., and Woychik, N.A. (1995) Six human RNA polymerase subunits functionally substitute for their yeast counterparts. Mol. Cell. Biol. 15, 6895-6900

40. Lim, C.Y., Santoso, B., Boulay, T., Dong, E., Ohler, U., Kadonaga, J.T. (2004) The MTE, a new core promoter element for transcription by RNA polymerase II. Genes Dev. 18, 1606-1617 